\title{
Inhibitory effects of B-cell translocation gene 2 on skin cancer cells via the Wnt/ $\beta$-catenin signaling pathway
}

\author{
SHOU-SONG GAO ${ }^{1}$, XIAO-HONG YANG ${ }^{2}$ and MENG WANG ${ }^{2}$ \\ ${ }^{1}$ Department of Cosmetic Surgery, Hangzhou Yue-ke Medical Cosmetology Clinic; ${ }^{2}$ Department of Dermatology, \\ First Affiliated Hospital of Zhejiang Chinese Medical University, Hangzhou, Zhejiang 310000, P.R. China
}

Received June 17, 2015; Accepted June 6, 2016

DOI: $10.3892 / \mathrm{mmr} .2016 .5596$

\begin{abstract}
B-cell translocation gene 2 (BTG2), a tumor suppressor gene, is downregulated in several types of human cancer cell. However, its function in skin cancer cells has not been fully elucidated. Therefore, the present study investigated the expression and function of BTG2 in skin cancer cells, and investigated the underlying molecular mechanism. The results indicated that BTG2 expression was downregulated in skin cancer cell lines. Overexpression of BTG2 significantly inhibited cell proliferation, cell cycle progression, and the invasion and migration of skin cancer cells. Furthermore, it was determined that overexpression of BTG2 significantly decreased the protein expression levels of $\beta$-catenin, cyclin D1 and v-myc avian myelocytomatosis viral oncogene homolog in skin cancer cells. This suggests that BTG2 may function as a tumor suppressor by interfering with the Wnt/ $\beta$-catenin signaling pathway in skin cancer cells. Thus, novel therapeutic strategies and agents targeting BTG2 may be potential treatments for skin cancer.
\end{abstract}

\section{Introduction}

As the largest organ of the body, the skin is frequently exposed to sunlight and ultraviolet rays. The function of skin is to protect internal organs from environmental harm and to maintain homeostasis (1), thus, the skin requires protection from frequent exposure to ultraviolet radiation, which may cause skin cancer. Skin cancer, the most common type of cancer worldwide, is a global public health problem and a burden on healthcare expenditures $(2,3)$. Therefore, more effective therapeutic strategies are required for its treatment.

B-cell translocation gene 2 (BTG2) is an important member of the BTG/TOB family $(4,5)$. Located on band 2, region 3 of the long arm of chromosome 1, BTG2 encodes a 158-amino

Correspondence to: Dr Meng Wang, Department of Dermatology, First Affiliated Hospital of Zhejiang Chinese Medical University, 45 Youdian Road, Hangzhou, Zhejiang 310000, P.R. China

E-mail: meng_wanghz@163.com

Key words: B-cell translocation gene 2, skin cancer, proliferation, cell cycle, invasion acid protein and structural deletions or changes often result in the occurrence of various tumors in humans (6-8). Previous studies have determined that BTG2 is a tumor suppressor of various cancer cells $(4,5)$. Zhang et al (9) reported that BTG2 suppressed proliferation and invasion of MDA-MB-231 triple-negative breast cancer cells. Additionally, BTG2 inhibited bladder cancer invasion via suppression of DNA methyltransferase 1 (10). BTG2 may also induce cellular differentiation, counteract cellular transformation and promote activity of pro-apoptotic stimuli (11-13). Additionally, BTG2 expression is frequently downregulated or blocked in various human tumors, including gastric and breast cancer $(14,15)$. However, the function of BTG2 in skin cancer remains unclear.

The Wnt $/ \beta$-catenin signaling pathway is important in the progression of tumors, including skin cancer (16-18). It was reported that mouse skin tumors demonstrated cytoplasmic and nuclear accumulation of $\beta$-catenin, and upregulation of $\beta$-catenin/Tcf target genes, including v-myc avian myelocytomatosis viral oncogene homolog and c-jun (19). Thus, inhibiting the Wnt/ $\beta$-catenin signaling pathway may prevent the progression of skin cancer.

The present study investigated the expression and function of BTG2 in skin cancer cells, as well as the underlying molecular mechanism. The results demonstrated that BTG2 was downregulated in skin cancer cells lines. Furthermore, overexpression of BTG2 significantly inhibited cell proliferation, cell cycle progression, cell invasion and migration of skin cancer cells via the Wnt/ $\beta$-catenin signaling pathway.

\section{Materials and methods}

Cell lines and culture. The human skin cancer cell lines (A431 and SCC13) were obtained from Shanghai Institutes for Biological Sciences, Chinese Academy of Sciences (Shanghai, China). All the cells were cultured as monolayers to $80 \%$ confluence in Dulbecco's modified Eagle's medium (DMEM; Invitrogen; Thermo Fisher Scientific, Inc., Waltham, MA, USA) supplemented with $10 \%$ heat-inactivated fetal bovine serum (FBS; Gibco; Thermo Fisher Scientific, Inc.) and $100 \mu \mathrm{g} / \mathrm{ml}$ penicillin-streptomycin (GE Healthcare, Logan, UT, USA) in a humidified incubator with $5 \% \mathrm{CO}_{2}$ at $37^{\circ} \mathrm{C}$.

Plasmids and transfection assays. A431 and SCC13 cell lines were transfected with the cDNA of the BTG2 gene. For stable 
transfection, pcDNA3.1 expression vector (Thermo Fisher Scientific, Inc.) was used for insertion of BTG2 cDNA between the KpnI and BamHI sites. The orientation of the insertion was confirmed via restriction digestion and DNA sequencing. Lipofectamine 2000 (Invitrogen; Thermo Fisher Scientific, Inc.) was used to transfect the pcDNA3.1-BTG2 expression vector or the empty pcDNA3.1 vector into A431 and SCC13 cells, in accordance with the manufacturer's protocol. The cells were then cultured in DMEM containing $800 \mu \mathrm{g} / \mathrm{ml}$ G418 for antibiotic selection. After 28 days, positive clones were collected from the A431 and SCC13 cell cultures in the pcDNA3.1-BTG2 vector transfection group (A431-BTG2 and SCC13-BTG2) and the empty pcDNA3.1 vector transfection group (A431-PC and SCC13-PC). Western blot analysis was performed in order to detect $\mathrm{BTG} 2$ protein expression in the A431-BTG2, A431-PC, SCC13-BTG2 and SCC13-PC cell groups.

Cell proliferation assay. Transfected and untreated cells were seeded into 96-well culture plates (Corning Life Sciences, MA, USA) at a density of $1 \times 10^{4}$ cells/well and cultured for $24 \mathrm{~h}$ with saturated humidity and $5 \% \mathrm{CO}_{2}$ at $37^{\circ} \mathrm{C}$ to form a cell monolayer. MTT solution (100 $\mu \mathrm{l}$; Nalge Nunc International, Roskilde, Denmark) was aliquoted per well and incubated with $5 \% \mathrm{CO}_{2}$ at $37^{\circ} \mathrm{C}$ for $4 \mathrm{~h}$. Next, the DMEM was discarded and $150 \mu \mathrm{l}$ dimethyl sulfoxide was added to each well. The absorbance was measured at $570 \mathrm{~nm}$ with a microplate reader (BD Biosciences, San Jose, CA, USA).

Cell cycle assay. When the cells reached 70-90\% confluence, a serum-free DMEM was applied for synchronization. The cells were cultured for $24 \mathrm{~h}$, then trypsinized (Sigma-Aldrich, St. Louis, MO, USA) and fixed with $100 \%$ ethanol overnight, followed by propidium iodide staining. Cell cycle analysis was performed after $30 \mathrm{~min}$ using a flow cytometer (BioVision, Inc., CA, USA). Each experiment was replicated three times.

Cell invasion and migration assays. Twenty-four well Transwell chambers ( $8 \mu \mathrm{m}$; Corning Life Sciences) were used to detect the invasive and migratory ability of the skin cancer cell lines. For the invasion assay, the upper chamber was washed with serum-free DMEM three times, filled with $20 \mu 1$ diluted Matrigel (BD Biosciences, Franklin Lakes, NJ, USA) and incubated for $30 \mathrm{~min}$ at $37^{\circ} \mathrm{C}$ to form the artificial basement membrane. Subsequently, all the cells were suspended in the serum-free DMEM and added to the upper chamber. The lower chamber was filled with DMEM containing $10 \%$ FBS. The cells were incubated for $24 \mathrm{~h}$ and those remaining in the upper chamber surface of the basement membrane were removed with cotton swabs, cells invading the lower chamber surface were stained with crystal violet. The number of cells crossing the polycarbonate membrane was counted under a microscope (CX22; Olympus Corporation, Tokyo, Japan), at a magnification of $x 400$. The migration assay was performed according to the above-mentioned procedure, except that Matrigel was not used, instead the Transwell chamber was used alone.

Western blotting. The cells were lysed using a lysis buffer (Sigma-Aldrich). The total protein $(30 \mu \mathrm{g})$ was separated from each group by $10 \%$ SDS-PAGE (Bio-Rad Laboratories, Inc., Hercules, CA, USA) and transferred onto polyvinylidene fluoride membranes (Thermo Fisher Scientific, Inc.). The membrane was blocked at room temperature for $1 \mathrm{~h}$ with Tris-buffered saline (TBS) containing 5\% non-fat milk and then incubated overnight at $4^{\circ} \mathrm{C}$ with the mouse primary antibodies (all from Santa Cruz Biotechnology, Dallas, TX, USA) against BTG2 (1:2,500; cat. no. sc-517187), $\beta$-catenin $(1: 2,000$; cat. no. sc-53484), cyclin D1 (1:3,000; cat. no. sc-20044), v-myc avian myelocytomatosis viral oncogene homolog (c-Myc; 1:2,500; cat. no. sc-47694) and $\beta$-actin (1:1,500; cat. no. sc-130300). After washing with TBS Tween-20 (TBST), the membrane was incubated for $1 \mathrm{~h}$ at $37^{\circ} \mathrm{C}$ with horseradish peroxidase-conjugated goat anti-mouse polyclonal antibody (1:3,000; Santa Cruz Biotechnology, Inc.; cat. no. 395760). Subsequent to another wash with TBST, protein expression was detected using an enhanced chemiluminescence kit (Bio-Rad Laboratories, Inc.)

Statistical analysis. Statistical analysis of experimental data was performed with SPSS 17.0 statistical analysis software (SPSS, Inc., Chicago, IL, USA). The Student's t-test was conducted for the comparison of two groups and one way analysis of variance was performed for multiple comparisons. Data are presented as the mean \pm standard deviation and $\mathrm{P}<0.05$ was considered to indicate a statistically significant difference.

\section{Results}

BTG2 expression in A431 and SCC13 cell lines and their transfectants. BTG2 protein expression in A431 and SCC13 cells and their transfectants was detected by western blot analysis. BTG2 protein expression was observed in A431-BTG2 and SCC13-BTG2 cells; however, it was not detected in A431, A431-PC, SCC13 and SCC13-PC cells (Fig. 1).

Effects of BTG2 overexpression on cell proliferation. The proliferation of A431-BTG2 cells was significantly reduced in comparison with the A431-PC and untreated A431 cells $(\mathrm{P}<0.05)$. No significant difference was identified between the control groups (A431 and A431-PC; Fig. 2A). Cell proliferation in the SCC13-BTG2 group was significantly reduced when compared with the control groups $(\mathrm{P}<0.05$; Fig. $2 \mathrm{~B})$.

Effects of BTG2 overexpression on cell cycle progression. Cell cycle analysis of A431 cells in each group was performed using flow cytometry. BTG2 overexpression significantly increased the number of A431 and SCC13 cells in the $\mathrm{G}_{0} / \mathrm{G}_{1}$ phase; however, it decreased the number of A431 and SCC13 cells in the $G_{2} / M$ phase $(P<0.05$; Fig. 3$)$. These results suggested that cell cycle distribution in A431 and SCC13 cells was affected by increased BTG2 expression levels and resulted in cell cycle arrest in the $G_{0} / G_{1}$ phase in A431 and SCC13 cells.

Effects of BTG2 overexpression on cell invasion and migration. The effects of BTG2 overexpression on invasion and migration of A431 and SCC13 cells were investigated. As shown in Fig. 4A and B, the invasive and migratory abilities of A431-BTG2 cells were significantly reduced when compared with A431-PC and untreated A431 cells $(\mathrm{P}<0.05)$. The effects 
A

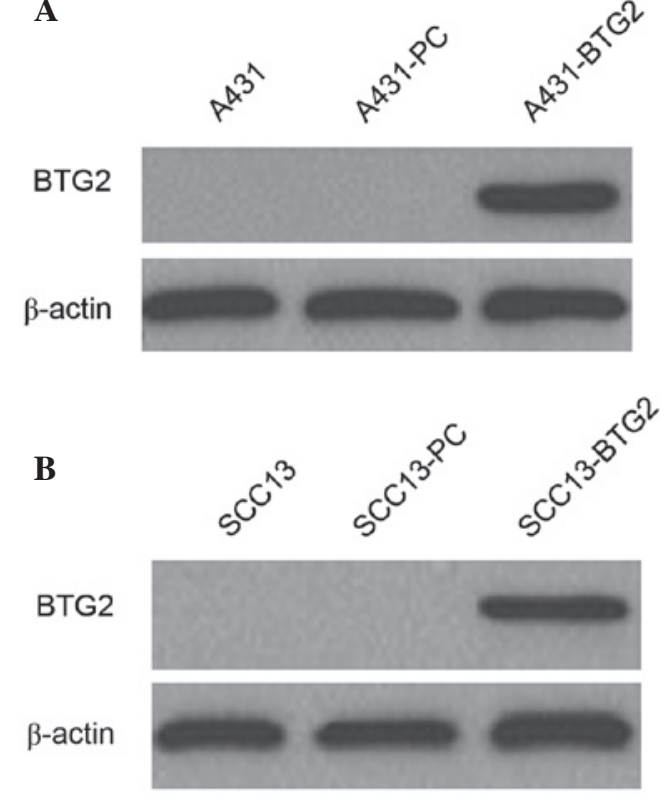

Figure 1. BTG2 expression in A431 and $\mathrm{SCC} 13$ cell lines and their transfectants. Western blot results of BTG2 in (A) A431, A431-PC, and A431-BTG2 and (B) SCC13, SCC13-PC and SCC13-BTG2 cells. BTG2, B-cell translocation gene 2; PC, empty vector.
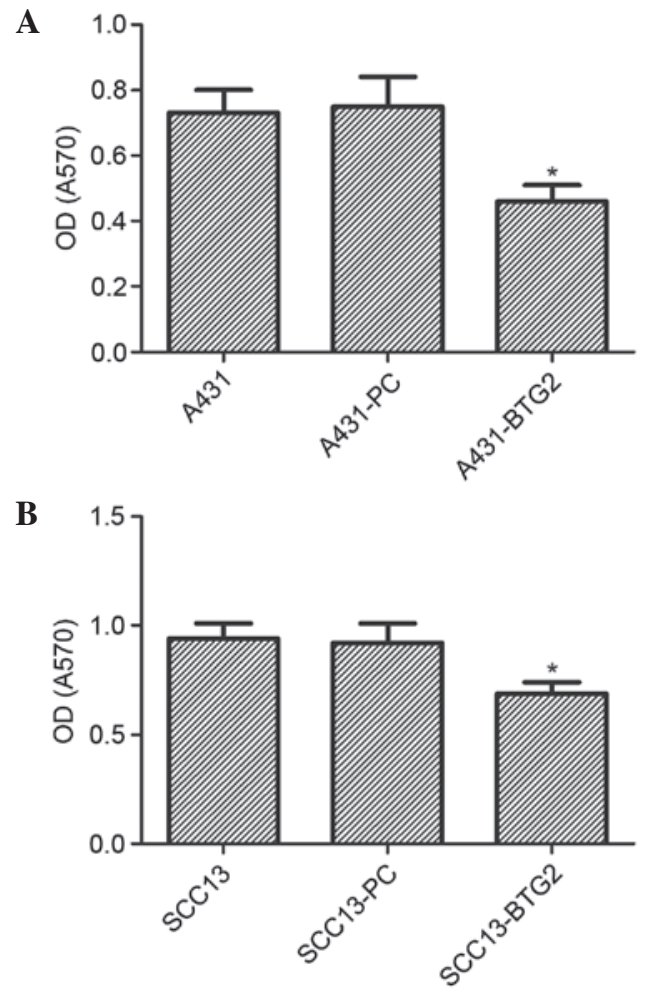

Figure 2. Effects of BTG2 overexpression on cell proliferation. MTT assay was performed to determine the effects of BTG2 overexpression in skin cancer cell proliferation. (A) Cell proliferation of A431-BTG2 cells was significantly decreased in comparison with A431 and A431-PC cells. (B) Cell proliferation of SCC13-BTG2 cells was significantly decreased when compared with $\mathrm{SCC} 13$ and SCC13-PC cells. ${ }^{*} \mathrm{P}<0.05$ vs. the control group. $\mathrm{BTG} 2$, B-cell translocation gene 2; PC, empty vector; OD, optical density.

of BTG2 overexpression on the invasion and migration of SCC13 cells were also determined. As shown in Fig. 4C and D,
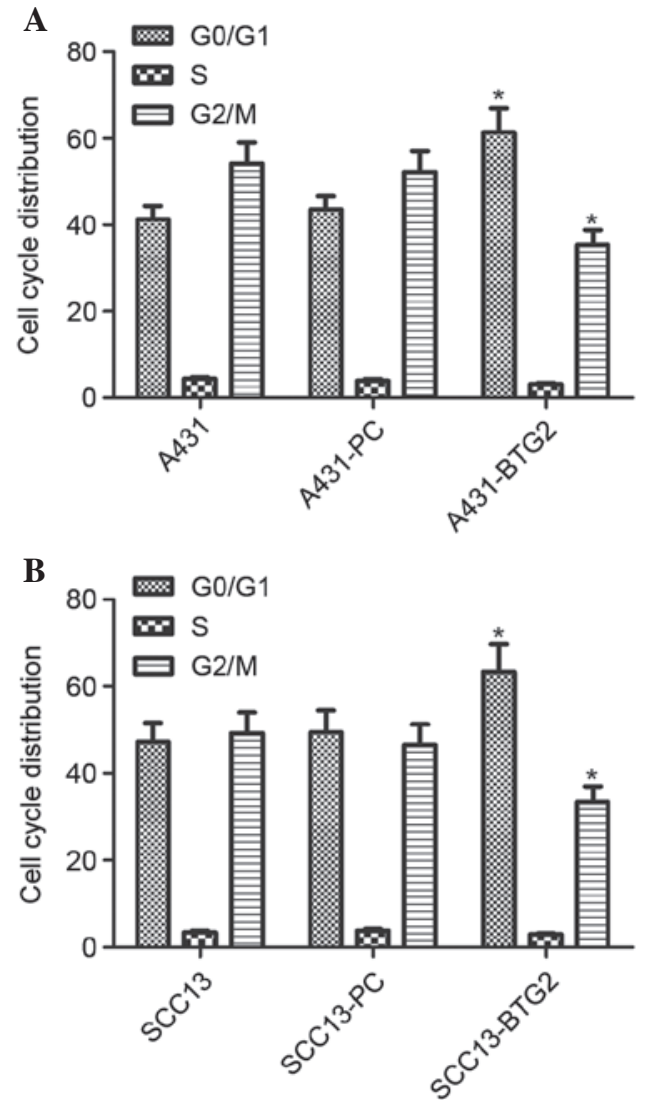

Figure 3. Effects of BTG2 overexpression on cell cycle progression. Flow cytometry was used to analyze the cell cycle distribution of A431 and $\mathrm{SCC} 13$ cells in each group. BTG2 overexpression increased the number of (A) A431-BTG2 cells at the $\mathrm{G}_{0} / \mathrm{G}_{1}$ phase, however, decreased cells in the $\mathrm{G}_{2} / \mathrm{M}$ phase, in comparison with A431 and A431-PC cells. Similar increases and decreases were found in the number of (B) SCC13-BTG2 cells at the $\mathrm{G}_{0} / \mathrm{G}_{1}$ and $\mathrm{G}_{2} / \mathrm{M}$ phase, respectively in comparison with $\mathrm{SCC} 13$ and $\mathrm{SCC} 13-\mathrm{PC}$ cells. ${ }^{\mathrm{P}}<0.05$ vs. the control group. BTG2, B-cell translocation gene 2; PC, empty vector.

invasive and migratory abilities of SCC13-BTG2 cells were significantly reduced when compared with SCC13-PC and untreated SCC13 cells $(\mathrm{P}<0.05)$.

Effects of BTG2 overexpression on $\beta$-catenin, cyclin D1 and $c$-Myc expression levels. Western blot analysis was performed to detect the effects of BTG2 overexpression on $\beta$-catenin, cyclin D1 and c-Myc. A significant decrease in the expression levels of $\beta$-catenin, cyclin D1 and c-Myc in A431-BTG2 cells when compared with A431-PC and untreated A431 cells $(\mathrm{P}<0.05$; Fig. 5).

\section{Discussion}

BTG2, a transient early response protein, has been reported to be a potential tumor suppressor gene $(6,12,20,21)$. Previous studies have highlighted the importance of BTG2 in tumor incidence, development, metastasis and invasion $(14,22,23)$. BTG2 overexpression may suppress proliferation of prostate cancer cells $(24,25)$. Additionally, BTG2 has frequently been downregulated or dysfunctional in previous studies of cancerous tumors $(6,26,27)$. Takahashi et al (23) determined that low expression levels of BTG2 in breast cancer may inhibit 
A
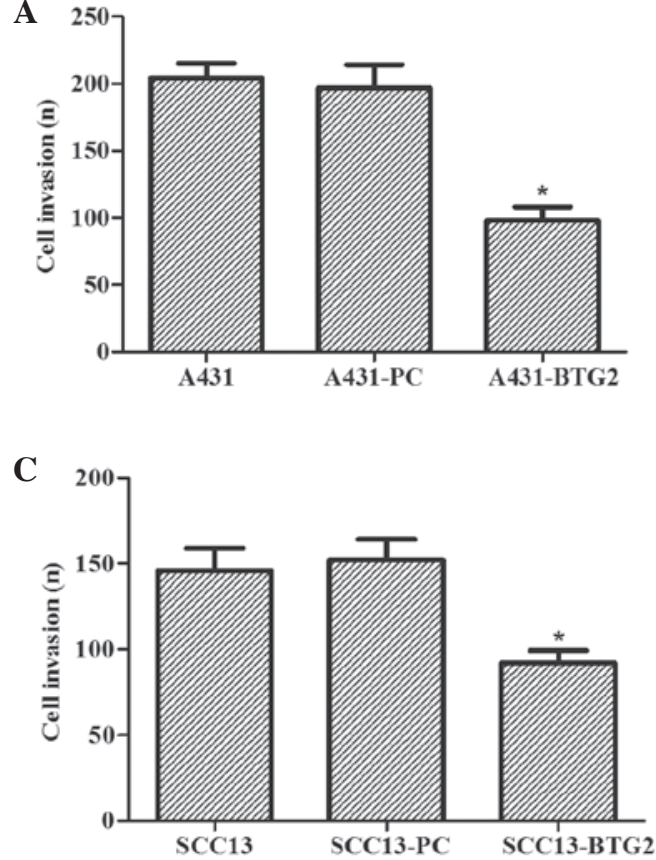

B

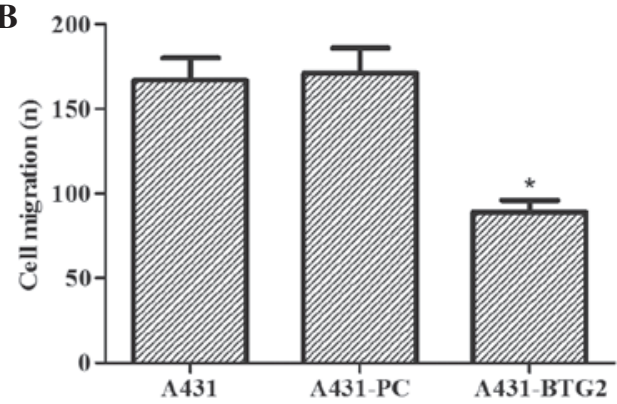

D

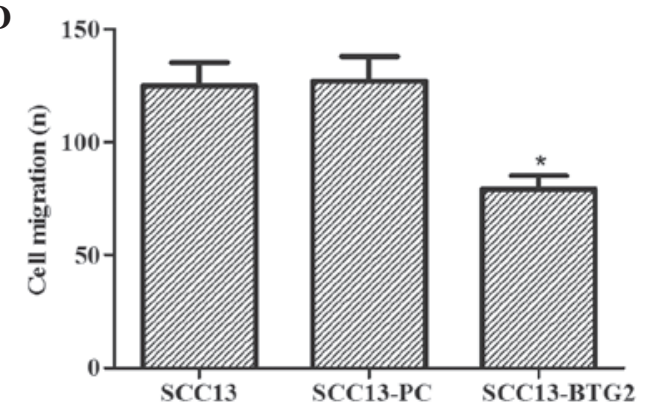

Figure 4. Effects of BTG2 overexpression on cell invasion and migration. Transwell chambers were used to determine the effect of BTG2 overexpression on invasion and migration of A431 and SCC13 cells. BTG2 overexpression decreased the quantity of A431-BTG2 cells (A) invading and (B) migrating through the membrane, in comparison with A431 and A431-PC cells. SCC13-BTG2 cell (C) invasion and (D) migration through the membrane compared with SCC13 and SCC13-PC cells. ${ }^{*} \mathrm{P}<0.05$ vs. the control group. BTG2, B-cell translocation gene 2; PC, empty vector.

A

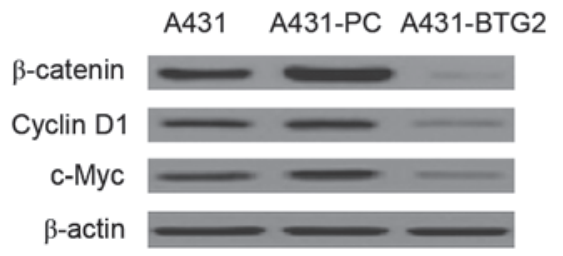

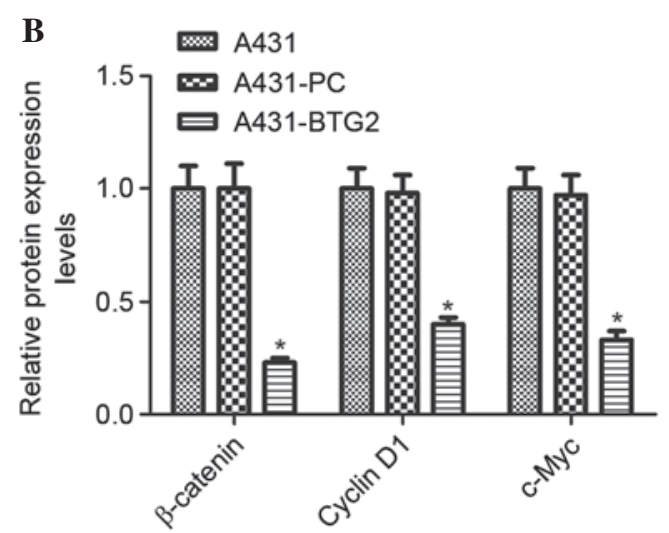

Figure 5. Effects of BTG2 overexpression on $\beta$-catenin, cyclin D1 and c-Myc. (A) Western blotting visualization of protein expression of $\beta$-catenin, cyclin D1 and c-Myc in A431, A431-PC and A431-BTG2 cells. (B) Quantification of relative protein expression of $\beta$-catenin, cyclin D1 and c-Myc in A431, A431-PC and A431-BTG2 cells. "P<0.05 vs. the control group. BTG2, B-cell translocation gene 2; c-Myc, v-myc avian myelocytomatosis viral oncogene homolog.

tumor metastasis (23). These studies indicated the association between BTG2 and tumor progression, and the importance of BTG2 in tumor incidence and proliferation. However, whether BTG2 is expressed in skin cancer and the effects BTG2 exerts on skin cancer remained unclear.

The present study investigated the expression and function of BTG2 in skin cancer progression and BTG2 expression was not observed in the skin cancer cell lines. Additionally, upregulation of BTG2 markedly inhibited cell proliferation, cell cycle progression, cell invasion and migration of skin cancer cells.

The cell cycle assay performed in the current study indicated that BTG2 overexpression may significantly increase the number of cells in the $G_{0} / G_{1}$ phase and decrease those in the $S$ and $G_{2}$ phases. This was consistent with the results of the cell proliferation assay performed in the present study, indicating that BTG2 exerted an inhibitory effect on cell proliferation. These results demonstrated the possible association between BTG2 overexpression and cell proliferation inhibition.

The effects of BTG2 on invasion and migration of skin cancer cells was examined using a Transwell assay. There was a significant decrease in cells crossing the polycarbonate membrane of the Transwell chamber, suggesting that BTG2 overexpression may be associated with the inhibition of skin cancer cell invasion and migration.

The present study demonstrated that overexpression of BTG2 significantly decreased the expression levels of $\beta$-catenin, cyclin D1 and c-Myc. The Wnt signaling pathway is fundamental for the determination of cell fate, polarity, proliferation and death (28). Additionally, environmental and 
genetic perturbations of the Wnt signaling pathway may lead to a variety of human diseases including various types of cancer (29). $\beta$-catenin, and its accumulation within the nucleus and cytoplasm, is associated with various types of cancer and is important in the successful functioning of Wnt signaling (30). In the current study, $\beta$-catenin was highly expressed in skin cancer cells and BTG2 overexpression significantly inhibited its expression levels. As target genes of $\beta$-catenin, cyclin D1 and c-Myc are powerful proto-oncogenes with similar downstream effects (31) and the two are associated with cell cycle control $(32,33)$. The present study identified a significant decrease in their expression levels, which may explain the inductive effect of BTG2 on the cell cycle arrest at the $G_{0} / G_{1}$ phase in skin cancer cells.

In conclusion, the present study investigated the effects of BTG2 overexpression on skin cancer cells and evaluated the underlying molecular mechanism. The results demonstrated that BTG2 was not expressed in skin cancer cells lines and its upregulation may suppress cell proliferation, cell cycle progression, cell invasion and migration of skin cancer cells via the $\mathrm{Wnt} / \beta$-catenin signaling pathway. Therefore, BTG2 may serve as a potential target for the treatment of skin cancer.

\section{References}

1. Kumar A, Shrestha PR, Pun J, Thapa P, Manandhar M and Sathian B: Profile of skin biopsies and patterns of skin cancer in a tertiary care center of Western Nepal. Asian Pac J Cancer Prev 16: 3403-3406, 2015.

2. Jiang YJ and Bikle DD: LncRNA: A new player in $1 \alpha, 25(\mathrm{OH})(2)$ vitamin $\mathrm{D}(3) / \mathrm{VDR}$ protection against skin cancer formation. Exp Dermatol 23: 147-150, 2014.

3. Singh T and Katiyar SK: Green tea polyphenol, (-)-epigallocatechin-3-gallate, induces toxicity in human skin cancer cells by targeting $\beta$-catenin signaling. Toxicol Appl Pharmacol 273 $418-424,2013$.

4. Ikematsu N, Yoshida Y, Kawamura-Tsuzuku J, Ohsugi M, Onda M, Hirai M, Fujimoto J and Yamamoto T: Tob2, a novel anti-proliferative Tob/BTG1 family member, associates with a component of the CCR4 transcriptional regulatory complex capable of binding cyclin-dependent kinases. Oncogene 18: 7432-7441, 1999.

5. Buanne P, Corrente G, Micheli L, Palena A, Lavia P, Spadafora C, Lakshmana MK, Rinaldi A, Banfi S, Quarto M, et al: Cloning of PC3B, a novel member of the PC3/BTG/TOB family of growth inhibitory genes, highly expressed in the olfactory epithelium. Genomics 68: 253-263, 2000.

6. Lim IK: TIS21 (/BTG2/PC3) as a link between ageing and cancer: Cell cycle regulator and endogenous cell death molecule. J Cancer Res Clin Oncol 132: 417-426, 2006.

7. Winkler GS: The mammalian anti-proliferative BTG/Tob protein family. J Cell Physiol 222: 66-72, 2010.

8. Duriez C, Moyret-Lalle C, Falette N, El-Ghissassi F and Puisieux A: BTG2, its family and its tutor. Bull Cancer 91: E242-E253, 2004.

9. Zhang Y-J, Wei L, Liu M, Li J, Zheng Y-Q, Gao Y and Li X-R: BTG2 inhibits the proliferation, invasion, and apoptosis of MDA-MB-231 triple-negative breast cancer cells. Tumor Biol 34: 1605-1613, 2013

10. Devanand P, Kim SI, Choi YW, Sheen SS, Yim H, Ryu MS, Kim SJ, Kim WJ and Lim IK: Inhibition of bladder cancer invasion by Sp1-mediated BTG2 expression via inhibition of DNA methyltransferase 1. FEBS J 281: 5581-5601, 2014.

11. el-Ghissassi F, Valsesia-Wittmann S, Falette N, Duriez C, Walden PD and Puisieux A: BTG2 (TIS21/PC3) induces neuronal differentiation and prevents apoptosis of terminally differentiated PC12 cells. Oncogene 21: 6772-6778, 2002.

12. Boiko AD, Porteous S, Razorenova OV, Krivokrysenko VI, Williams BR and Gudkov AV: A systematic search for downstream mediators of tumor suppressor function of p53 reveals a major role of BTG2 in suppression of Ras-induced transformation. Genes Dev 20: 236-252, 2006.
13. Lim YB, Park TJ and Lim IK: B cell translocation gene 2 enhances susceptibility of HeLa cells to doxorubicin-induced oxidative damage. J Biol Chem 283: 33110-33118, 2008.

14. Zhang L, Huang H, Wu K, Wang M and Wu B: Impact of BTG2 expression on proliferation and invasion of gastric cancer cells in vitro. Mol Biol Rep 37: 2579-2586, 2010.

15. Möllerström E, Kovács A, Lövgren K, Nemes S, Delle U, Danielsson A, Parris T, Brennan DJ, Jirström K, Karlsson P and Helou K: Up-regulation of cell cycle arrest protein BTG2 correlates with increased overall survival in breast cancer, as detected by immunohistochemistry using tissue microarray. BMC Cancer 10: 296, 2010.

16. Li J, Ji L, Chen J, Zhang W and Ye Z: Wnt/ß-catenin signaling pathway in skin carcinogenesis and therapy. Biomed Res Int 2015: 964849, 2015.

17. Kuphal S and Bosserhoff AK: Phosphorylation of $\beta$-catenin results in lack of $\beta$-catenin signaling in melanoma. Int J Oncol 39: 235-243, 2011.

18. Kang MI, Baker AR, Dextras CR, Cabarcas SM, Young MR and Colburn NH: Targeting of noncanonical Wnt5a signaling by AP-1 blocker dominant-negative Jun when it inhibits skin carcinogenesis. Genes Cancer 3: 37-50, 2012.

19. Bhatia N and Spiegelman VS: Activation of Wnt/beta-catenin/Tcf signaling in mouse skin carcinogenesis. Mol Carcinog 42: 213-221, 2005.

20. Zhang Z, Chen C, Wang G, Yang Z, San J, Zheng J, Li Q, Luo X, Hu Q, Li Z and Wang D: Aberrant expression of the p53-inducible antiproliferative gene BTG2 in hepatocellular carcinoma is associated with overexpression of the cell cycle-related proteins. Cell Biochem Biophys 61: 83-91, 2011.

21. Horvilleur E, Bauer M, Goldschneider D, Mergui X, de la Motte A, Bénard J, Douc-Rasy S and Cappellen D: p73alpha isoforms drive opposite transcriptional and post-transcriptional regulation of MYCN expression in neuroblastoma cells. Nucleic Acids Res 36: 4222-4232, 2008.

22. Yang CH, Yue J, Pfeffer SR, Handorf CR and Pfeffer LM: MicroRNA miR-21 regulates the metastatic behavior of B16 melanoma cells. J Biol Chem 286: 39172-39178, 2011.

23. Takahashi F, Chiba N, Tajima K, Hayashida T, Shimada T, Takahashi M, Moriyama H, Brachtel E, Edelman E, Ramaswamy $\mathrm{S}$ and Maheswaran S: Breast tumor progression induced by loss of BTG2 expression is inhibited by targeted therapy with the ErbB/HER inhibitor lapatinib. Oncogene 30: 3084-3095, 2011.

24. Segev DL, Kucirka LM, Oberai PC, Parekh RS, Boulware LE, Powe NR and Montgomery RA: Age and comorbidities are effect modifiers of gender disparities in renal transplantation. J Am Soc Nephrol 20: 621-628, 2009.

25. Liu M, Wu H, Liu T, Li Y, Wang F, Wan H, Li X and Tang H: Regulation of the cell cycle gene, BTG2, by miR-21 in human laryngeal carcinoma. Cell Res 19: 828-837, 2009.

26. Hagan S, Al-Mulla F, Mallon E, Oien K, Ferrier R, Gusterson B, García JJ and Kolch W: Reduction of Raf-1 kinase inhibitor protein expression correlates with breast cancer metastasis. Clin Cancer Res 11: 7392-7397, 2005.

27. Kawakubo H, Brachtel E, Hayashida T, Yeo G, Kish J, Muzikansky A, Walden PD and Maheswaran S: Loss of B-cell translocation gene-2 in estrogen receptor-positive breast carcinoma is associated with tumor grade and overexpression of cyclin d1 protein. Cancer Res 66: 7075-7082, 2006.

28. Saito-Diaz K, Chen TW, Wang X, Thorne CA, Wallace HA, Page-McCaw A and Lee E: The way Wnt works: Components and mechanism. Growth Factors 31: 1-31, 2013.

29. MacDonald BT, Tamai $\mathrm{K}$ and $\mathrm{He} \mathrm{X}$ : Wnt/beta-catenin signaling: Components, mechanisms, and diseases. Dev Cell 17: 9-26, 2009.

30. Wang J, Wang X, Gong W, Mi B, Liu S and Jiang B: Increased expression of beta-catenin, phosphorylated glycogen synthase kinase 3 beta, cyclin D1, and c-myc in laterally spreading colorectal tumors. J Histochem Cytochem 57: 363-371, 2009.

31. Ripple MJ, Parker Struckhoff A, Trillo-Tinoco J, Li L, Margolin DA, McGoey R and Del Valle L: Activation of c-Myc and Cyclin D1 by JCV T-Antigen and $\beta$-catenin in Colon Cancer. PLoS One 9: e106257, 2014.

32. Baldin V, Lukas J, Marcote M, Pagano M and Draetta G: Cyclin $\mathrm{D} 1$ is a nuclear protein required for cell cycle progression in G1. Genes Dev 7: 812-821, 1993.

33. Dang CV: c-Myc target genes involved in cell growth, apoptosis, and metabolism. Mol Cell Biol 19: 1-11, 1999. 